\title{
A Monolithic PDMS Waveguide System Fabricated Using Soft-Lithography Techniques
}

\author{
David A. Chang-Yen, Member, IEEE, Richard K. Eich, Member, IEEE, and Bruce K. Gale, Member, IEEE
}

\begin{abstract}
A monolithic waveguide system using poly(dimethyl siloxane) (PDMS) was designed, fabricated, and characterized. The waveguide demonstrated good confinement of light and relatively low attenuation at $0.40 \mathrm{~dB} / \mathrm{cm}$. The robustness and handling properties of the completed waveguides were excellent, and the process yield exceeded $96 \%$. The waveguide did exhibit moderate temperature and humidity sensitivity but no temporal variation, and insertion loss remained stable over extended periods of time. Applications of this waveguide system in microscale sensing are immense, judging by the frequency of use of PDMS as the substrate for microfluidic and biomedical systems. The monolithic nature of the waveguides also reduces their cost and allows integration of optical pathways into existing PDMS-based microsystems.
\end{abstract}

Index Terms-Micro-total-analysis systems, poly(dimethyl siloxane), soft lithography, waveguide.

\section{INTRODUCTION}

$\mathbf{P}$ OLY(DIMETHLY SILOXANE) (PDMS) is commonly used to fabricate microscale fluidic systems, such as separation systems, micromixers, micropumps and valves, as well as diagnostic chips and DNA hybridization arrays [1]-[7]. Many microscale diagnostic systems rely upon light sensing methods and would benefit greatly from integrated optical conduction pathways. However, the flexible nature of PDMS does not support nonhomogeneous waveguides readily, as any relatively stiff or brittle waveguide that is embedded in the PDMS system would be prone to breakage upon handling. In addition, introducing a different waveguide material into an existing PDMS microsystem complicates the fabrication process and can lead to undesirable material and fabrication interactions. To avoid these issues, we have developed a technique to use PDMS as both the waveguide core and substrate material, thus creating a monolithic waveguide readily compatible with PDMS microfluidic systems. This system is extremely robust and is capable of withstanding the stresses and shock associated with rough handling that would severely damage more brittle waveguide systems. PDMS also has very high light transmittance $(>95 \%)$ over a large frequency range [8], does not require special handling procedures, and is relatively low

\footnotetext{
Manuscript received April 14, 2004; revised February 15, 2005. This work was supported in part by the University of Utah College of Engineering, Salt Lake City.

D. A. Chang-Yen is with the Department of Mechanical Engineering, University of Utah, Salt Lake City, UT 84112 USA, and also with Wasatch Microfluidics, Salt Lake City, UT 84123 USA (e-mail: dac10@utah.edu)

R. K. Eich is with the Department of Bioengineering, University of Utah, Salt Lake City, UT 84112 USA (e-mail: r.eich.utah.edu).

B. K. Gale is with the Department of Mechanical Engineering, University of Utah, Salt Lake City, UT 84112 USA (e-mail: gale@eng.utah.edu).

Digital Object Identifier 10.1109/JLT.2005.849932
}

cost. In addition, using the techniques described in this paper, one could readily produce an integrated microfluidic and microoptical system using only one molding or photolithography step.

Design of the integrated optics morphology, interfacing, and packaging in this work is based upon previous literature by Chang-Yen et al. [9]. In this study, the waveguide material used instead of SU-8 is the material PDMS, more commonly known as silicone. This material presents several advantages over SU-8 as a waveguide material: a rapid and repeatable mold fabrication process, higher optical transmittance, and increased robustness [10]-[15]. However, microscale PDMS structures have traditionally been fabricated using peel-off molds [16], [17]. Since the molded waveguides must have a lower refractive index than their substrate, a low-refractive-index (less than 1.45-1.47), flexible material that is capable of bonding to PDMS but not the mold is required. It was determined that the material that best fit these parameters was PDMS itself, necessitating a difference in refractive index between the waveguiding PDMS and the substrate PDMS.

For waveguiding to occur in a PDMS waveguide on a PDMS substrate, the waveguide core PDMS must possess a higher refractive index than the substrate. This requirement is based on premise that effective waveguiding will occur only when total internal reflection occurs at the core-substrate interface as the light propagates inside the core. The number of modes that the core can carry will increase with the size of the core, as well as the difference in core-substrate refractive indexes. For this paper, the size of the waveguide was fixed at $125 \mu \mathrm{m} \times$ $125 \mu \mathrm{m}$ in a cross section to closely match the diameter of the silica optical fiber used to couple the light in and out. Since the waveguide system was intended for biosensor applications with strong interactions of the light at the core-superstrate interface, high-order modes were preferable. Thus, the waveguide core fabrication process that was developed in this study hinged on the ability to produce the largest refractive-index difference between the core and substrate. This refractive-index difference was accomplished by modifying the curing process for the PDMS for the different components of the waveguide system.

\section{Methodology}

\section{A. Waveguide Design and Process Overview}

The waveguide system design was constrained by a number of factors: simplicity of fabrication, compatibility with microfluidics, robustness, coupling requirements, and modal characteristics. Using PDMS to fabricate a monolithic waveguide system produced an extremely robust waveguide system, 


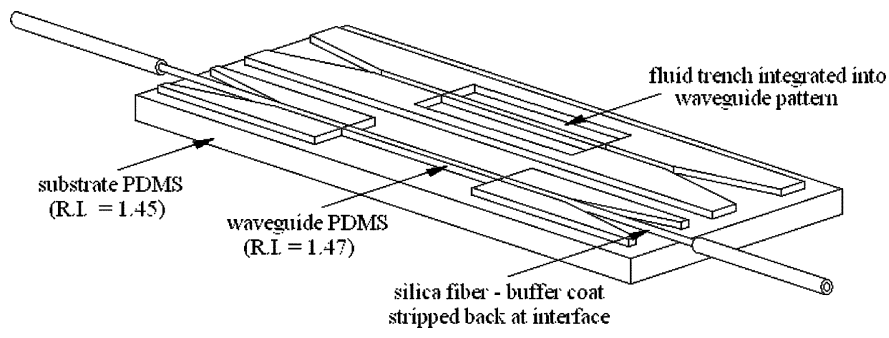

Fig. 1. PDMS waveguide with interface to silica fiber.

TABLE I

ELLIPSOMETRIC PDMS SAMPLES

\begin{tabular}{c|c|c}
\hline Sample & Curing Temp erature ("C) & Curing Perind \\
\hline 1 & $25(\mathrm{RT})$ & 48 hours \\
\hline 2 & 50 & 60 minutes \\
\hline 3 & 100 & 30 minutes \\
\hline 4 & 100 & 60 minutes \\
\hline 5 & 150 & 30 minutes \\
\hline 6 & 150 & 60 minutes \\
\hline
\end{tabular}

both from a manufacturing and a handling standpoint. The PDMS waveguides can be made in only a few steps, and microfluidic and packaging components can be integrated in the same steps. Since heat was used to differentiate the waveguide core and substrate regions, the fabrication process had to separate the PDMS formation of the core and substrate, a process not normally considered with traditionally PDMS demolding [17]. Previous work with SU-8 waveguides [9] used an integrated fiber-optic coupler, and this coupling method was included to maintain simple and low-cost operation of the waveguide system. As stated previously, a standard $125-\mu \mathrm{m}$ optical fiber was used to couple the waveguide to fiber-optic test instrumentation, which essentially fixed the cross-sectional dimensions of the waveguide. All of the design requirements were fulfilled by the waveguide design, and the system fabrication was achieved using a single mold over a period of approximately two days-most of which was curing time. A diagram of the fabricated and packaged waveguide is shown in Fig. 1.

\section{B. Refractive-Index Measurement}

PDMS can be obtained in a non-cross-linked form under the tradename SYLGARD 184 (Dow Corning), as a two-part resin and cross linker. Setting of the elastomer is achieved by mixing the two components in a 10:1 ratio by weight, respectively. The chemical cross-linking reaction takes place spontaneously at room temperature over an approximately 48 -hour period. This setting time can be drastically shortened by applying heat to the setting PDMS. Since the cross-linking effect appears to be greatly increased by heating, one of our hypotheses was that the refractive index of PDMS would increase with baking time and temperature. To determine if this hypothesis was true, a series of PDMS samples spun at $6100 \mathrm{r} / \mathrm{min}$ onto borosilicate glass substrates were tested using ellipsometry. Four samples were tested using the conditions shown in Table I. Measurements of refractive index were determined for wavelengths of 460 and $610 \mathrm{~nm}$ and plotted together. These specific wavelengths were chosen to match the excitation and emission wavelengths for a specific fluorescent dye used in previous literature [9] that is sensitive to changes in oxygen concentration. A MATLAB simulation model was also created based on the eigenvalue equations [18] to determine the mode confinement and mode peaks that the waveguide would be capable of carrying.

\section{Waveguide Fabrication}

1) Overview: The waveguides were developed to be part of an optical sensing system, and the fabrication procedure was developed using a combination of work in previous literature involving fabrication SU-8 waveguides [9] and the refractive-index measurements taken from the previously described ellipsometer test procedures. The PDMS patterns are created photolithographically using SU-8 molds, and the fluidic interfacing system is also PDMS-based, making the completed device, without external packaging, essentially monolithic.

2) SU-8 Mold Fabrication: The SU-8 mold fabrication process, though usually overlooked, posed sufficient challenges to warrant further elucidation. The SU-8 layer from which the mold was patterned was spun onto 3 -in silicon wafers to a height of $125 \mu \mathrm{m}$ to match the cladding diameter of the coupling fiber optics. SU-8 50 from Microchem was used as received, although the recommended soft-baking time of $30 \mathrm{~min}$ at $95{ }^{\circ} \mathrm{C}$ was altered to $21 / 2 \mathrm{~h}$ to remove as much of the solvent as possible before exposure. SU-8 on wafers that were baked for only $30 \mathrm{~min}$ buckled upon postexposure baking, whereas the longer soft-baked wafers produced smooth resist patterns. Following the development of the wafers, they were coated with a diluted detergent solution to prevent sticking of the PDMS to the mold. Treatment of the SU-8 molds with the fluorinating compound (tridecafluoro-1,1,2,2-tetrahydrooctyl) triethoxysilane (Gelest) proved unsuccessful, and the detergent solution proved to be the most reliable method to prevent sticking.

3) PDMS Molding: The waveguide fabrication was accomplished in two basic steps: filling of the SU-8 mold with PDMS and baking and then waveguide substrate casting. Liquid PDMS prepolymer was poured onto the detergent-treated SU-8 mold and allowed to flow completely into the pattern (Step 2). The excess PDMS that did not fill into the SU-8 pattern was scraped off carefully with a razor blade to bring the PDMS level to the top of the SU-8 mold (Step 3). The wafer was baked at $150{ }^{\circ} \mathrm{C}$ for $60 \mathrm{~min}$ to cure the PDMS at a refractive index of 1.47. The baked wafer was then cooled to room temperature, and more PDMS prepolymer was poured over the entire wafer and allowed to cure at room temperature to a refractive index of 1.45 (Step 4). The substrate layer when cured was bonded to the core, allowing the entire structure to be peeled off the mold as a single piece (Step 5). The waveguides were then ready for packaging and testing. This fabrication process is presented in Fig. 2.

\section{Waveguide Packaging}

The PDMS waveguides were coupled to $50 \mu \mathrm{m}$ core $/ 125 \mu \mathrm{m}$ cladding silica fiber (Thorlabs) using a novel monolithic taper interface. Instrument interfacing with the silica fiber was made using SubMiniature Version A (SMA)-type connections (Thorlabs). Alignment of the silica fiber with the PDMS waveguide 


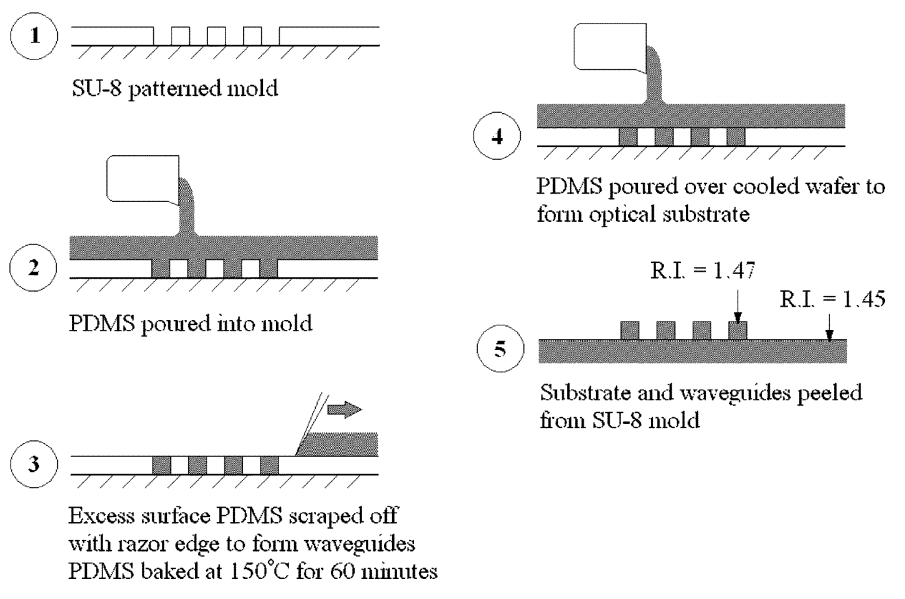

Fig. 2. PDMS waveguide fabrication process.

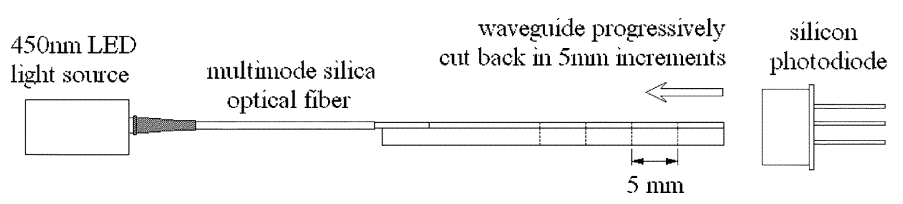

Fig. 3. Attenuation apparatus.

was made automatically upon insertion into the interface, as shown in the lower waveguide in Fig. 1.

\section{E. Waveguide Attenuation}

To determine the attenuation loss per unit length of the PDMS waveguides, a cutback test was performed (Fig. 3). A 70-mm waveguide was created with a fiber-optic taper interface at one end for 460-nm light injection. The other end of the waveguide was cut flat with a razor blade to allow the light to exit directly to a silicon photodiode (Thorlabs). To prevent substrate modes from interfering with the detected signal, an absorbing plate was used to cover the exit face of the substrate region (not shown). The light source used was an USB-LS-450 LED module (Oceanoptics). Dark readings were taken before each light measurement to minimize the effect of thermal noise and ambient light interference. Light intensity measurements were taken for waveguide lengths of 70 to $25 \mathrm{~mm}$ by progressively cutting off 5-mm lengths from the emission end of the waveguide. The voltage output from the photodiode was converted to a light intensity value using a calibration curve provided by Thorlabs and converted to attenuation measurements using [19]

$$
A=-10 \log \left(\frac{P_{\text {out }}}{P_{\text {in }}}\right)
$$

where $A$ is the attenuation in decibels (dB), $P_{\text {in }}$ was the power injected into the waveguide, and $P_{\text {out }}$ was the power measured by the photodiode. $P_{\text {in }}$ was measured by injecting light from the bare optical fiber into the photodiode. The results were plotted to determine the attenuation per unit length (in decibels per centimeter, $\mathrm{dB} / \mathrm{cm}$ ).

\section{F. Temporal Stability}

It was unknown if any differences in refractive index of PDMS created by the different curing procedures would be

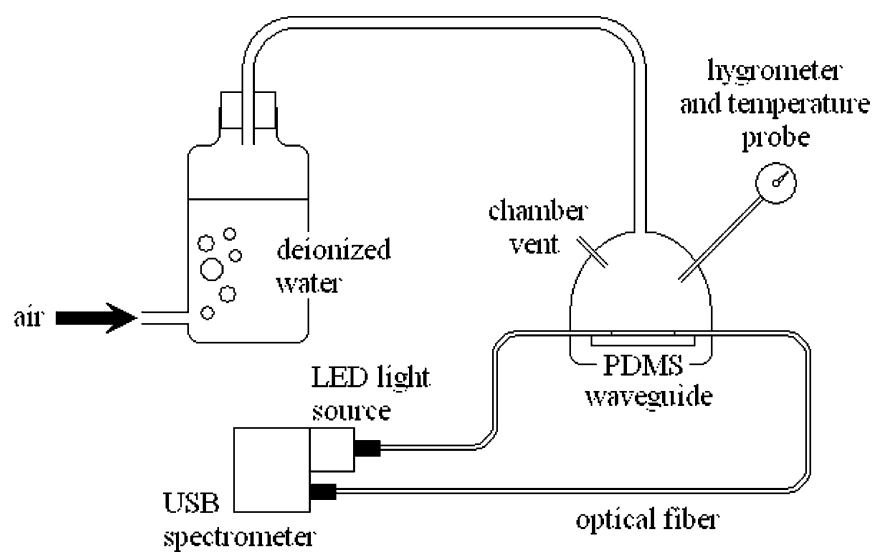

Fig. 4. Controlled humidity test apparatus.

stable over a long period of time. PDMS treated with oxygen plasmas to create a hydrophilic surface have been observed to revert to a hydrophobic surface over a period of approximately $2 \mathrm{~h}$. This reversion is thought to be caused by migration of un-cross-linked low-molecular weight PDMS chains to the surface [20]-[22]. Since the proposed reason that the changes in the refractive index of PDMS is based upon degrees of cross linking, it was hypothesized that un-cross-linked low-molecular weight PDMS chains from within the substrate would eventually migrate into the core, lowering the refractive index. If this presumed migration does take place within the waveguide samples, the period of time required to cause a drift in waveguide loss could be much longer than $2 \mathrm{~h}$, as considerable polymer migration would be necessary to significantly alter the waveguide refractive-index profile and cause a measurable change in loss. As such, transmittance of a $3.5-\mathrm{cm}$ waveguide was measured over a period of 30 days to determine the rate of drift, if any.

\section{G. Thermal and Relative Humidity Sensitivity}

Since the PDMS waveguide fabrication procedure used differences in curing temperature to define the core and substrate regions, it was theorized that the PDMS waveguides may exhibit some sensitivity to changes in temperature. In addition, relative humidity $(\mathrm{RH})$ could possible affect the transmittance of the waveguide due to the relatively low waveguide core refractive index. To test thermal effects, a 6.5-cm-long PDMS waveguide was created and interfaced at both ends to optical fibers as described previously. The waveguide was then exposed to temperatures varying from $30{ }^{\circ} \mathrm{C}$ to $70{ }^{\circ} \mathrm{C}$, while measuring the optical transmittance at $460 \mathrm{~nm}$. Relative humidity was tested in a controlled RH chamber shown in Fig. 4. The relative humidity was varied in the chamber by progressively allowing air to flow through the water bubbler to saturate the waveguide test chamber atmosphere with water vapor. Once the chamber had reached a stable maximum $\mathrm{RH}(85 \%)$, the water was emptied from the bubbler, and dry air was allowed to flow into the test chamber, gradually forcing out the humid air. Temperature readings in the waveguide test chamber were taken concurrently to ensure that thermal effects were not influencing the procedure. During the course of the entire test, the chamber temperature was limited to a temperature variation of not more than $1{ }^{\circ} \mathrm{C}$. 
TABLE II

PDMS REFRACTIVE- INDEX CHANGES FOR VARYING CURING CONDITIONS

\begin{tabular}{|c|c|c|c|c|}
\hline \multirow[t]{2}{*}{ Sample } & \multirow{2}{*}{$\begin{array}{l}\text { Curing Temperature } \\
\left({ }^{\circ} \mathrm{C}\right)\end{array}$} & \multirow{2}{*}{$\begin{array}{l}\text { Curing } \\
\text { Period }\end{array}$} & \multicolumn{2}{|c|}{ Refractive index } \\
\hline & & & $460 \mathrm{~nm}$ & $610 \mathrm{~nm}$ \\
\hline 1 & $25(\mathrm{RT})$ & 48 hours & 1.451 & 1.416 \\
\hline 2 & 50 & 60 minutes & 1.465 & 1.417 \\
\hline 3 & 100 & 30 minutes & 1.465 & 1.422 \\
\hline 4 & 100 & 60 minutes & 1.466 & 1.421 \\
\hline 5 & 150 & 30 minutes & 1.469 & 1.432 \\
\hline 6 & 150 & 60 minutes & 1.472 & 1.432 \\
\hline
\end{tabular}

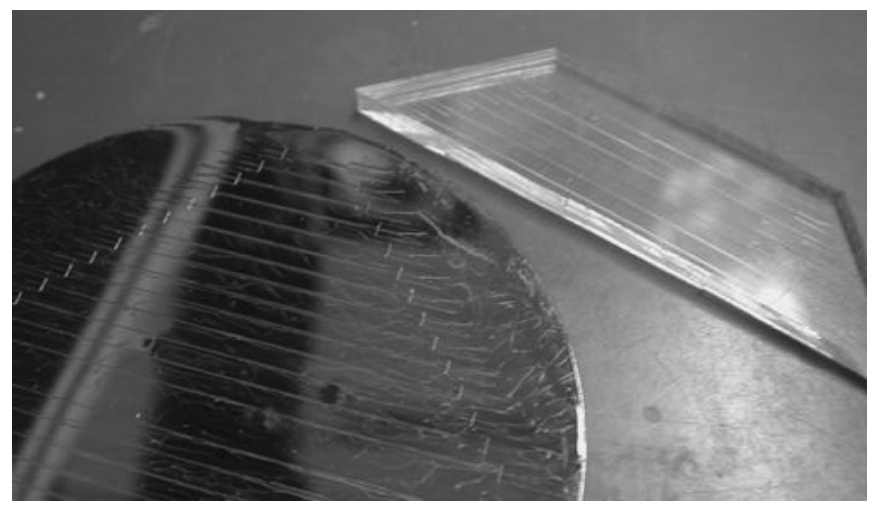

Fig. 5. PDMS waveguides and SU-8 mold.

\section{RESUlTS AND DISCUSSION}

\section{A. Ellipsometry}

Before the waveguide fabrication procedure was developed and implemented (Fig. 2), the ellipsometric data was reviewed to determine whether the hypothesis that varied heat curing would change the refractive index of PDMS was true. The results are shown in Table II. Comparing samples 1 and 6, the refractive index of light at both 460 and $610 \mathrm{~nm}$ demonstrated a change of 0.02 , which should be sufficient for waveguiding. A MATLAB simulation of electrical field confinement yielded $609 E^{y}$ peaks and $304 E^{x}$ peaks at $460 \mathrm{~nm}$ and $459 E^{y}$ peaks and $229 E^{x}$ peaks at $610 \mathrm{~nm}$, where $E^{x}$ and $E^{y}$ are the electrical field strengths in the horizontal and vertical direction, respectively. Thus, the observed increase in refractive index would allow limited multimode waveguiding to occur and justified the next stage of the study of waveguide fabrication.

\section{B. Waveguide Fabrication}

A set of completed PDMS waveguides is shown in Fig. 5 next to its corresponding SU-8 mold. Dual copies of each waveguide were made in each mold to improve the chances for successful waveguide fabrication, although on average, of the 28 waveguides on each mold, only a single guide failed to form successfully, demonstrating a 96\% process yield. Fig. 6 shows a single $6.5-\mathrm{cm}$ waveguide that has been separated from the complete batch and interfaced to silica optical fibers. To observe if confinement was actually taking place in the waveguide, a single 6-cm waveguide was interfaced to an optical fiber at one end and cut flat at the other end with a razor blade for imaging. Fig. 7 shows an optical microscope image of the 460-nm light

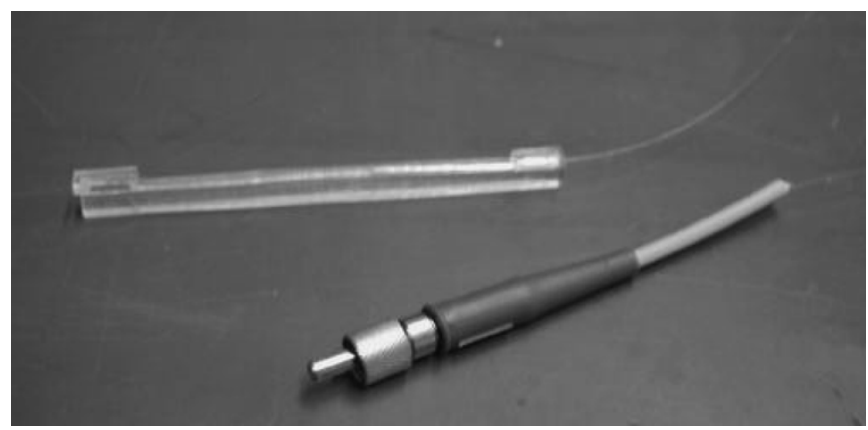

Fig. 6. PDMS waveguide interfaced to silica fiber.

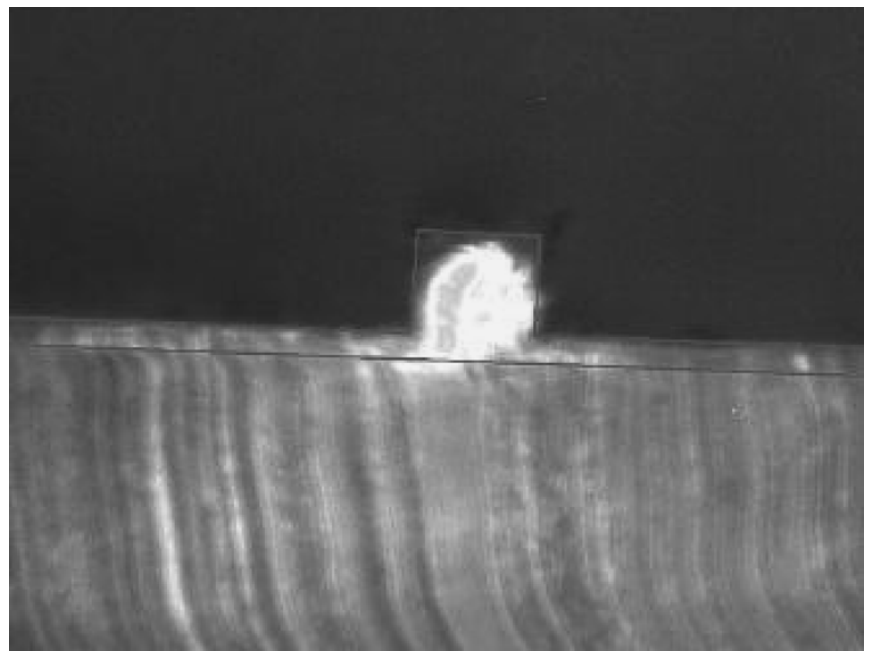

Fig. 7. Microscope end face image of PDMS waveguide.

pattern at the cut end face of the waveguide. The high-refractive-index region of the PDMS is outlined for clarity. Note that in Fig. 7, due to the imperfect removal of PDMS from the top surface of the SU-8 mold during Step 3 of the fabrication process (Fig. 2), a thin layer of high-refractive-index PDMS (approximately $25 \mu \mathrm{m}$ ) can be seen at the top surface of the substrate. This thin layer acted as a planar waveguide, and laterally escaping light in the horizontal direction can been seen clearly, particularly to the left of the core region. The vertical striations on the end face were caused by the razor blade used to cut the waveguide. Light can also be seen in the substrate region, although this light is believed to result from modes too high to be conducted by the waveguide escaping at the optical fiber interface, and being conducted to the end face by the substrate.

\section{Attenuation}

The cutback procedure used to measure the attenuation of the waveguide demonstrates a loss of approximately $0.40 \mathrm{~dB} / \mathrm{cm}$ (Fig. 8). This value is comparable to existing polymeric optical waveguide materials, such as SU-8 and Norland Optical Adhesive 73 [11], [12]. The large attenuation shown on the $y$-scale of Fig. 8 can be attributed to the $P_{\text {in }}$ value used for the attenuation calculation. The power exiting directly from the silica fiber was injected into the photodiode; thus, the cumulative intensity of all modes supported by the fiber were measured as $P_{\text {in }}$. However, using MATLAB calculations of the PDMS waveguide modal 


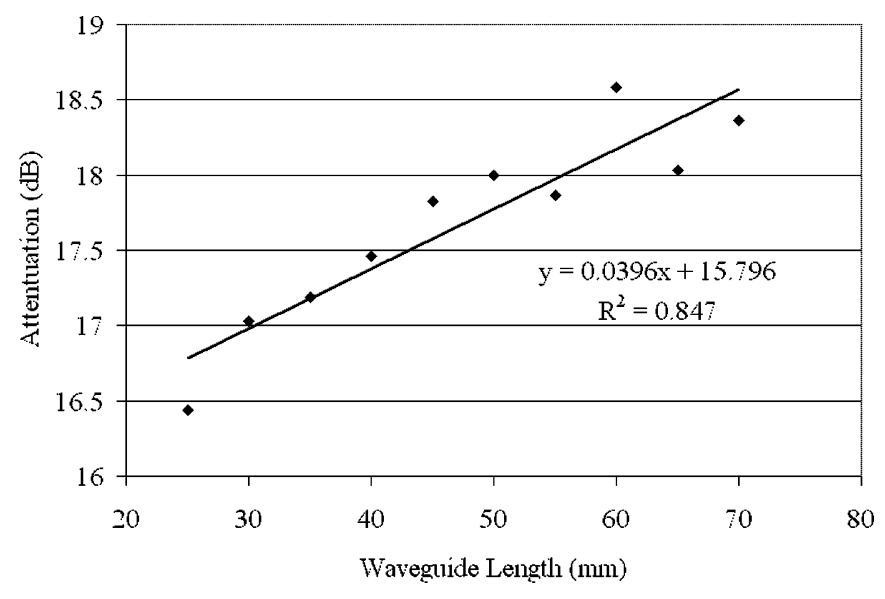

Fig. 8. PDMS waveguide attenuation at $460 \mathrm{~nm}$.

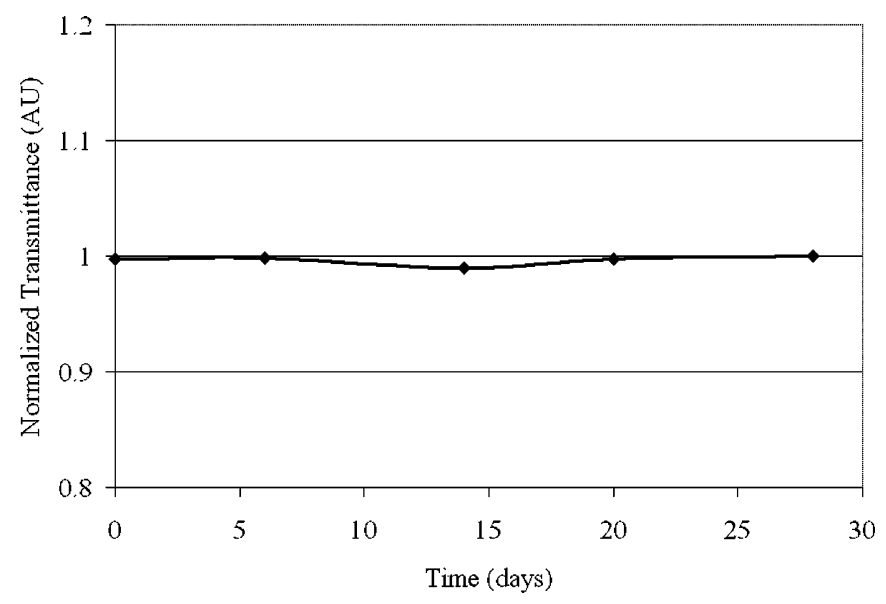

Fig. 9. PDMS waveguide temporal stability.

support and numerical aperture values given for the silica fiber, it was determined that a large number of modes that are supported by the fiber are not supported by the waveguide. These modes exit the waveguide as radiation modes, occurring approximately in the first $640 \mu \mathrm{m}$ of the waveguide. These losses were verified with microscope inspection. The scattering of the plot can be attributed to minor misalignments of the waveguide to the photodiode following cutback, as well as loss at the end face caused in major part by scattering by the observed striations of the cut face and in minor part by Fresnel reflection.

\section{Temporal Stability}

Over a 30-day period, the maximum measured drift of normalized transmittance (Fig. 9) was approximately 1.05\% and disappeared as time passed. The typical variation in time was less than $0.3 \%$. Comparing this drift to all the previous data, the results indicate that the variation can be attributed to transducer noise. This inference is supported particularly well by the relative humidity sensitivity data (not shown), where values varied by more than $1.5 \%$ for several data points. Thus, the waveguides appear to be relatively stable long term, and any molecular rearrangement in the PDMS is insufficient to reduce or eliminate the refractive-index differences created during curing. Temporal stability at higher-than-average temperatures and relative humidity have not yet been determined, and further study is re- quired to determine whether long exposures to either condition bear lasting effects.

\section{E. Thermal and Relative Humidity Sensitivity}

Results from the temperature sensitivity testing showed an approximately linear dependence, up to approximately $15 \%$, although the effects were not observed to be permanent. The humidity testing also demonstrated a similar level of temporary sensitivity as temperature, although the dependence was nonlinear. Only speculation can be made on the cause of the apparent thermal and RH dependence, but the changes were judged to be significant in both cases. Despite observed repeatability of the dependence, further testing is required to determine its exact nature and cause.

\section{CONCLUSION}

A monolithic poly(dimethyl siloxane) (PDMS) waveguide system was successfully fabricated using soft-lithography rapid-prototyping methods. Attenuation of the waveguides was comparable with current micropatterned polymeric waveguide systems, and temporal stability was excellent. The waveguides demonstrated a degree of sensitivity to temperature and relative humidity (approximately 15\% in each case), the characteristics of which require further study and indicate that the waveguides could possibly be used as sensors for temperature and humidity. Completed waveguides were extremely robust, and the manufacturing process yield exceeded $96 \%$. The combination of these exceptional handling characteristics with the lost cost, low toxicity, biocompatibility, and commonness of PDMS as a microsystem substrate material supports the applicability of this waveguide system for integration into microanalysis systems and other commercial devices.

\section{REFERENCES}

[1] A. Papra, A. Bernard, D. Juncker, N. B. Larsen, B. Michael, and E. Delamarche, "Microfluidic networks made of poly(dimethylsiloxane), $\mathrm{Si}$, and Au coated with polyethylene glycol for patterning proteins onto surfaces," Langmuir, vol. 17, pp. 4090-4095, Jun. 2001.

[2] S. L. Walker, S. Bhattacharjee, E. M. V. Hoek, and M. Elimelech, "A novel asymmetric clamping cell for measuring streaming potential of flat surfaces," Langmuir, vol. 18, pp. 2193-2198, Mar. 2002.

[3] D. J. Beebe, J. S. Moore, Q. Yu, R. H. Liu, M. L. Kraft, B.-H. Jo, and C. Devadoss, "Microfluidic tectonics: a comprehensive construction platform for microfluidic systems," Proc. Nat. Academy Sci., vol. 97, pp. 13 488-13 493, Dec. 2000.

[4] O. Hofmann, G. Voirin, P. Niedermann, and A. Manz, "Three-dimensional microfluidic confinement for efficient sample delivery to biosensor surfaces. Application to immunoassays on planar optical waveguides," Anal. Chem., vol. 74, pp. 5243-5250, Oct. 2002.

[5] R. A. Vijayendran, K. M. Motsegood, D. J. Beebe, and D. E. Leckband, "Evaluation of three-dimensional micromixer in a surface-based biosensor," Langmuir, vol. 19, pp. 1824-1828, Mar. 2003.

[6] P. A. Auroux, D. Iossifidis, D. R. Reyes, and A. Manz, "Micro total analysis systems. 2. Analytical standard operations and applications," Anal. Chem., vol. 74, pp. 2637-2652, Jun. 2002.

[7] H. Chen, D. Acharya, A. Gajraj, and J.-C. Meiners, "Robust interconnects and packaging for microfluidic elastomeric chips," Anal. Chem., vol. 75, pp. 5287-5291, Oct. 2003.

[8] G. S. Rajan, G. S. Sur, J. E. Mark, D. W. Schaefer, and G. Beaucage, "Preparation and application of some unusually transparent poly(dimethylsiloxane) nanocomposites," J. Polym. Sci., B, vol. 41, pp. 1897-1901, Aug. 2003.

[9] D. A. Chang-Yen and B. K. Gale, "An integrated optical oxygen sensor fabricated using rapid-prototyping techniques," Lab-on-a-Chip, vol. 3, pp. 297-301, Jun. 2003. 
[10] K. B. Mogensen, J. El-Ali, A. Wolff, and J. P. Kutter, "Integration of polymer waveguides for optical detection in microfabricated chemical analysis systems," Appl. Opt., vol. 42, pp. 4072-4079, Jul. 2003.

[11] X.-M. Zhao, S. P. Smith, S. J. Waldman, G. M. Whitesides, and M. Prentiss, "Demonstration of waveguide couplers fabricated using microtransfer molding," Appl. Phys. Lett., vol. 71, pp. 1017-1019, Aug. 1997.

[12] J.-S. Kim, J.-W. Kang, and J.-J. Kim, "Simple and low cost fabrication of thermally stable polymeric multimode waveguides using a UV-curable epoxy," Jpn. J. Appl. Phys., vol. 42, pp. 1277-1279, Mar. 2003.

[13] A. Borreman, S. Musa, A. A. M. Kok, M. B. J. Diemeer, and A. Driessen, "Fabrication of polymeric multimode waveguides and devices in SU-8 photoresist using selective polymerization," in Proc. 2002 Symp. IEEE/LEOS Benelux Chapter, 2002, pp. 83-86.

[14] Y. Ansel, D. Grau, and M. Holzki, "Assembly of optical fibers for the connection of polymer-based waveguide," in Proc. SPIE, vol. 4947, 2003, pp. 102-112.

[15] C.-H. Lin, G.-B. Lee, S.-H. Chen, and G.-L. Chang, "Micro capillary electrophoresis chips integrated with buried SU-8/SOG optical waveguides for bio-analytical applications," Sens. Actuators A, Phys., vol. 107, pp. $125-131$, Oct. 2003.

[16] P. Thiébaud, L. Lauer, W. Knoll, and A. Offenhäusser, "PDMS device for patterned application of microfluids to neuronal cells arranged by microcontact printing," Biosens. Bioelec., vol. 17, pp. 87-93, Jan. 2002.

[17] J. R. Anderson, D. T. Chiu, R. J. Jackman, O. Cherniavskaya, J. C. McDonald, H. Wu, S. H. Whitesides, and G. M. Whitesides, "Fabrication of topologically complex three-dimensional microfluidic systems in PDMS by rapid prototyping," Anal. Chem., vol. 72, pp. 3158-3164, Jul. 2000.

[18] R. G. Hunsperger, Integrated Optics: Theory and Technology, ser. Springer Series in Optical Sciences. Berlin, Heidelberg, GDR: Springer-Verlag, 1982, vol. 33, pp. 16-42.

[19] D. K. Mynbaev and L. L. Scheiner, Fiber-Optic Communications Technology. Englewood Cliffs, NJ: Prentice-Hall, 2001, pp. 54-55.

[20] H. C. Hillborg, Loss and Recovery of Hydrophobicity of Polydimethylsiloxane After Exposure to Electrical Discharges. Solna, Sweden: Nykopia Global Print AB, 2001, pp. 13-15.

[21] S. D. Gillmor, B. J. Larson, J. M. Braun, C. E. Mason, L. E. Cruz-Barba, F. Denes, and M. G. Lagally, "Low-contact-angle polydimethyl siloxane (PDMS) membranes for fabricating micro-bioarrays," in Proc. 2nd Annu. Int. IEEE-EMBS Special Topic Conf. Microtechnologies Medicine Biology, 2002, pp. 51-56.

[22] E. Delamarche, C. Donzel, F. S. Kamounah, H. Wolf, M. Geissler, R. Stutz, P. Schmidt-Winkel, B. Michel, H. J. Mathieu, and K. Schaumburg, "Microcontact printing using poly(dimethylsiloxane) hydrophilized by poly(ethylene oxide) silanes," Langmuir, vol. 19, pp. 8749-8758, Oct. 2003.

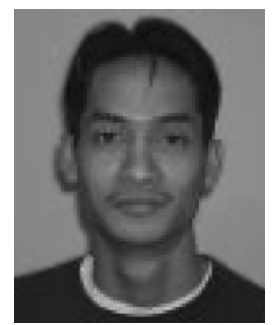

David A. Chang-Yen (M'03) was born in Trinidad, West Indies, on August 30, 1977. He received the B.Sc. and M.Sc. degrees in biomedical engineering from Louisiana Tech University, Ruston, in May 2000 and August 2002, respectively, and the Ph.D. degree in mechanical engineering from the University of Utah, Salt Lake City, in May 2005.

$\mathrm{He}$ is currently a Research Associate with the Department of Mechanical Engineering at the University of Utah. He is also Vice-President for Research and Development for Wasatch Microfluidics, Salt Lake City, UT. He is the author of two journal articles on polymeric-based sensors and nanotechnology and is the coapplicant on six related technology disclosures and one patent.

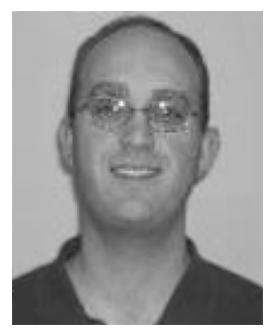

Richard K. Eich (M'03) received the B.S. degree in electronics engineering technology from Brigham Young University, Provo, UT, in 2002 and is currently working toward the M.S. degree in bioengineering from the University of Utah, Salt Lake City. His thesis work involves implementing the extraction and amplification of DNA on a nanoporous aluminum oxide membrane contained within a poly(dimethyl siloxane) (PDMS)-based microfluidic system.

His research interests include electronic instrumentation related to the field of biology and medicine.

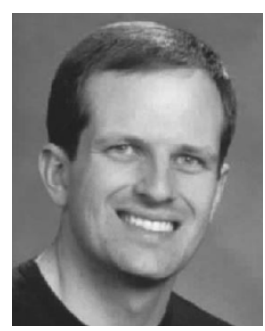

Bruce K. Gale (S'98-A'00-M'03) received the B.S. degree in mechanical engineering from Brigham Young University, Provo, UT, in 1995 and the Ph.D. degree in bioengineering from the University of Utah, Salt Lake City, in 1999.

He spent over two years as an Assistant Professor of biomedical engineering at Louisiana Tech University, Ruston, and the Institute for Micromanufacturing. In December 2001, he joined the Mechanical Engineering Department at the University of Utah as an Assistant Professor. His interests include medical- and biological-based applications of microfluidics, and his work has recently involved micromachined particle separation systems and detectors, microarray manufacturing methods, and sensors related to these applications. 